\title{
Wireless Power Transfer Through Simultaneous Near-Field Focusing and Far-Field Synthesis
}

\author{
Rafael G. Ayestarán, Senior Member, IEEE, Germán León, Member, IEEE, Marcos R. Pino, Paolo \\ Nepa, Member, IEEE
}

\begin{abstract}
A technique for the synthesis of antenna arrays that can account simultaneously for Near-Field Focusing on one or more spots and Far-Field specifications is proposed, to deal with Wireless Power Transfer applications. The technique is based on the minimization of a properly defined cost function accounting for both Nearand Far-Field requirements at the same time, and including a trade-off parameter to balance each specification in the final result. By synthesizing the weights that have to be applied to the elements of the array fulfilling all the specifications, a wireless link between the antenna array and different devices may be established more efficiently, since power radiated at undesired positions or directions can be reduced. Some illustrative examples are presented to show the ability of the method to solve problems with independent specifications for Near- and Far-Field distributions. A prototype of a $16 \times 16$ planar array operating at $16 \mathrm{GHz}$ has been realized and characterized; it is shown that it is able to focus on two Near-Field spots and at an assigned Far-Field direction, simultaneously.
\end{abstract}

Index Terms-Near-Field Focusing, Far-Field pattern synthesis, array optimization, antenna arrays, wireless power transfer.

\section{INTRODUCTION}

$\mathbf{N}$ EAR Field Focusing (NFF) [1]-[4] is a wellknown technique typically used to concentrate the radiated field around an assigned point (focal point) in the Near-Field (NF) region of a radiating system. Antenna arrays (or other array technologies such as reflectarrays or transmittarrays) have been mostly used due to their ability to shape the antenna near field by using a proper set of weights applied to each element [2], [3]. NFF has been employed in applications such as medical hyperthermia [5]-[7], non-contact microwave inspection [8], RFID [9]-[11], imaging, etc. It is suitable for short-range communications between close devices

This work was partially supported by the Spanish Ministerio de Ciencia, Innovacion y Universidades under the project TEC201786619-R (ARTEINE), by the Gobierno del Principado de Asturias under project GRUPIN-IDI-2018-000191, and by the Mobility Grants of the University of Oviedo financially supported by Banco Santander. R. G. Ayestarán, G. León and M. R. Pino are with the University of Oviedo, Department of Electrical Engineering, Gijón, Asturias, 33203 Spain (e-mail: \{rayestaran,gleon,mpino\}@uniovi.es).

P. Nepa is with the University of Pisa, Department of Information Engineering, Pisa, 56122, Italy (e-mail: paolo.nepa@unipi.it). with information about each other position, and avoiding interference from other elements that might be placed in the same environment, as in Internet of Things (IoT) or 5G scenarios, where Wireless Power Transfer (WPT) or Wireless Power and Information Transfer (WPIT) are becoming popular [12]-[15]. In WPT, the radiated field is concentrated on the device to be powered, avoiding waste of energy in other positions or directions of the space. NFF fits perfectly this objective as a tool able to establish an efficient power or data transfer from one device to another.

In most conventional NFF antenna design problems, the goal is to obtain the weights that have to be applied to the array elements so that it generates a NF distribution focused at an assigned position of the antenna NF region, typically (but not necessarily) in the broadside axis. For this kind of problem, the use of the ConjugatePhase (CP) method has been proven to be an excellent choice [2], [9]. It accounts for the relative position between each radiating element and the assigned focal point, and the different distance between the focal point and each radiating element is compensated by applying a phase shift to the feeding current of each element. It results that all the contributions arrive in-phase to the desired point creating a constructive interference (actually, the field peak is at a point between the focal point and the array surface, and such effect may be compensated by a proper choice of the assigned focal point [3]). This approach has been successful to solve many NFF problems. However, it is limited to one focal spot. If more focal points have to be accounted for, for example due to the presence of a set of devices that have to be included in the wireless link (WPT or WPIT are good examples of this situation), specific synthesis strategies have to be followed. Some methodologies used to overcome these limitations involve multiple-feed reflectarrays [13], ad-hoc microwave designs [16], [17], artificial neural networks [18], optimization approaches [19]-[22] or time-reversal techniques [21]-[23]. While the first two mentioned alternatives involve specific hardware design, optimization approaches have been shown to be an effective method for solving such problems from an algorithmic point of view, allowing the design 
of solutions for different radiating structures (arrays, reflectarrays, transmittarrays, etc.) and resulting in a flexible and powerful methodology.

Additionally, in many applications it is also necessary that the Far Field (FF) radiation pattern is controlled. For example, if NFF is used to communicate with a near device placed at the focal point, minimizing the FF radiation might result in reducing the total radiated power, and hence in a more efficient communication in terms of energy. This could become more important in scenarios where devices of interest might be placed in both the NF and FF regions. If data are also transmitted (WPIT), FF pattern control is useful in presence of interfering elements that might be distorting the communication from any unpredictable FF direction. Potentially, other applications might require different additional constraints on the FF radiation pattern while the array is still focused on the NF targets, or a simultaneous focusing on near spots and FF directions. In such cases, the FF pattern should be controlled or reduced, and a technique able to account simultaneously for both NF and FF specifications is required.

In this contribution, we propose a synthesis scheme able to account for constraints both in the NF and FF regions, simultaneously. The proposed technique allows to design radiating systems able to concentrate the field into one or more NF spots and FF directions, without wasting power or interfering with devices at other positions, and considering specifically wireless applications with multiple users in an indoor scenario (e.g. a meeting room), at microwaves or millimeter-waves band, where the use of arrays able to optimize the radiations towards a number of wireless devices is becoming a hot research topic [24]-[26]. The proposed technique is based on the direct minimization of a cost function designed to optimize the weights applied to an antenna array. It is a flexible method to perform synthesis in NFF antennas [19], [20], and can easily deal with almost arbitrary NF and FF specifications at the same time.

This paper is organized as follows. Section II states the formulation and main characteristics of the problem. Some illustrative examples and results are discussed in Section III, including the design of a prototype of an antenna array designed to deal with both $\mathrm{NF}$ and $\mathrm{FF}$ specifications, plus an example in a possible $5 \mathrm{G}$ scenario. Finally, some conclusions are outlined.

\section{Problem StATEMENT AND FORMULATiON}

The field radiated by the $N$ elements of an arbitrary array at a position $\vec{r}$ of the NF environment (outside the reactive near-field region) is given by:

$$
\vec{E}(\vec{r})=\sum_{n=1}^{N} w_{n} \vec{f}_{n}\left(\vec{r}, \overrightarrow{r_{n}^{\prime}}\right) \frac{e^{-j k\left|\vec{r}-\vec{r}_{n}^{\prime}\right|}}{\left|\vec{r}-\vec{r}_{n}^{\prime}\right|}
$$

where $\overrightarrow{r_{n}^{\prime}}$ is the position vector corresponding to the $n$-th element of the array, $w_{n} \in \mathbb{C}$ is the weight applied to this $n$-th element, $k=2 \pi / \lambda$ is the free space wavenumber, $\lambda$ is the free space wavelength, and $\vec{f}_{n}\left(\vec{r}, \overrightarrow{r_{n}^{\prime}}\right)$ is a vector accounting for the radiation pattern of the $n$-th element in the direction defined by both $\vec{r}$ and $\overrightarrow{r_{n}^{\prime}}$, which may be disregarded if isotropic elements are assumed. In most cases, the array is assumed to be uniformly distributed, for example in the $x-y$ plane, leading to a simple expression of the positions $\overrightarrow{r_{n}^{\prime}}$. Moreover, if the array is rectangular, $N=N_{x} \times N_{y}$, where $N_{x}$ is the number of elements in the $x$-axis and $N_{y}$ is the number of elements in the $y$-axis.

In a similar way, the FF radiation pattern produced by the array can be expressed as

$$
\vec{E}_{F F}(\theta, \phi)=\sum_{n=1}^{N} w_{n} \underline{\vec{f}}_{n}(\theta, \phi) e^{j \vec{k} \cdot \vec{r}_{n}^{\vec{r}}}
$$

where the direction of propagation is defined by the propagation vector $\vec{k}$, and hence by the angles $\theta$ and $\phi$, and $\vec{f}_{n}$ is a normalized version of the radiation pattern of the $n$-th element of the array, including the propagation factor and the corresponding attenuation.

A matrix-version of Eq. (1) may be set up by grouping the field samples at different positions of the environment onto a vector, so that

$$
\mathbf{e}_{N F}=\mathbf{S}_{N F} \cdot \mathbf{w}
$$

where $\mathbf{e}_{N F}=\left[E_{N F}^{c}\left(\overrightarrow{r_{1}}\right), E_{N F}^{c}\left(\overrightarrow{r_{2}}\right) \ldots E_{N F}^{c}\left(r_{M}\right)\right]^{T}$ is a column vector containing the sampled values of a field component or polarization (or combination of them, indicated with the $c$ superscript) radiated at the $M$ considered positions of the near-field region; $\mathbf{w}=$ $\left[w_{1}, w_{2}, \ldots, w_{N}\right]^{T}$ is a vector with the weights applied to each element of the array, $\mathbf{S}_{N F}$ is a matrix with elements $s_{N F}(n, m)=\vec{f}_{n}\left(\vec{r}_{m}, \overrightarrow{r_{n}^{\prime}}\right) \frac{e^{-j k\left|r_{m}-\vec{r}_{n}^{\prime}\right|}}{\left|r_{m}-r_{n}^{\prime}\right|}$; and $(.)^{T}$ stands for the transpose operator. It is interesting to notice that no approximation about the geometry of the array has been assumed, so that the elements might be placed at any positions $\overrightarrow{r_{n}^{\prime}}$ (at least from the algorithmic point of view).

Similarly, FF vector $\mathbf{e}_{F F}$ and matrix $\mathbf{S}_{F F}$ can be defined so that

$$
\mathbf{e}_{F F}=\mathbf{S}_{F F} \cdot \mathbf{w}
$$

with $\mathbf{e}_{F F}=\left[E_{F F}^{c}\left(\xi_{1}\right), E_{F F}^{c}\left(\xi_{2}\right) \ldots E_{F F}^{c}\left(\xi_{L}\right)\right]^{T}$ being a column vector containing the sampled values of the far field radiated towards the $L$ directions of the space $\xi_{l}=\{\theta, \phi\}_{l}, l=1 \ldots L$ normalized to remove dependence with the distance, $\mathbf{S}_{F F}$ is a matrix with elements $s_{F F}(n, l)=\vec{f}_{n}\left(\xi_{l}\right) e^{-j \vec{k}\left(\xi_{l}\right) \cdot r_{n}^{\vec{r}}}$, and $\vec{k}\left(\xi_{l}\right)$ is the propagation vector towards the direction $\xi_{l}$.

The problem of calculating the weights $\mathbf{w}$ can be stated as a minimization problem provided that a proper 
cost function is defined. A general cost function to be minimized is proposed so that the problem may be formulated as

$$
\underset{\mathbf{w}}{\operatorname{argmin}}\left\{f_{N F}(\mathbf{w})+\gamma f_{F F}(\mathbf{w})\right\}
$$

where $f_{N F}(\mathbf{w})$ and $f_{F F}(\mathbf{w})$ are generic functions of the weights applied to the array, that should be defined according to the NF and FF requirements for a specific application, while $\gamma$ is a trade-off parameter used to balance the importance of each term in the cost function. Above parameter is related to the level of the values of each term, and it is also influenced by the normalization of (2). Additionally, some constraints might be included in the optimization problem if requested by the specifications (resulting in a constrained optimization scheme).

As far as NF specifications are concerned, the first term might be designed to guarantee that the NF radiation is close to the desired NF distribution, with a maximum at the focal point and minimal values at any other position. It can be done by establishing a target NF distribution specified through a vector $\mathbf{e}_{N F}$ with null values except at the positions corresponding to the desired focal points, where a value 1 is set. Then, (5) becomes

$$
\underset{\mathbf{w}}{\operatorname{argmin}}\left\{\left\|\mathbf{e}_{N F}-\mathbf{S}_{N F} \cdot \mathbf{w}\right\|^{2}+\gamma f_{F F}(\mathbf{w})\right\}
$$

where $\|$.$\| is the l 2$-norm, and the square has been included to improve the convergence rate of the optimization schemes that might be used.

Notice that $f_{N F}(\mathbf{w})$ might be defined using another approach if the NF specifications of the problem were different. For example, if minimum transmitted power were required, but specific field levels were necessary at certain points, we might use $f_{N F}(\mathbf{w})=\|\mathbf{w}\|^{2}+$ $\left\|\tilde{\mathbf{e}}_{N F}-\tilde{\mathbf{S}}_{N F} \cdot \mathbf{w}\right\|^{2}$, where the matrix $\tilde{\mathbf{S}}_{N F}$ is a reduced version of $\mathbf{S}_{N F}$ only accounting for the positions of the focal points, and $\tilde{\mathbf{e}}_{N F}$ containing the required values at each focal point; also, the first term would ensure the minimum field level at any other position, as it implies a minimization of the transmitted power. Once more, the squares have been included to help the convergence of the chosen optimization algorithms.

The function $f_{F F}(\mathbf{w})$ has to be defined according to the FF specifications. For example, let us consider the case aiming to provide a certain NF distribution and reducing the overall FF radiated power. For this problem, the proposed FF-specification function might be defined according to:

$$
f_{F F}(\mathbf{w})=\operatorname{var}\left\{\mathbf{S}_{F F} \cdot \mathbf{w}\right\}
$$

where var stands for the variance, leading to the following optimization problem:

$$
\underset{\mathbf{w}}{\operatorname{argmin}}\left\{\left\|\mathbf{e}_{N F}-\mathbf{S}_{N F} \cdot \mathbf{w}\right\|^{2}+\gamma \operatorname{var}\left\{\mathbf{S}_{F F} \cdot \mathbf{w}\right\}\right\}
$$

The second term minimizes the variance of the FF radiation, with respect to the observation direction, leading to a radiation pattern as omnidirectional as possible (namely, an antenna directivity as close as possible to unity). In a different application, a certain FF radiation pattern $\mathbf{e}_{F F}$ might be specified, so that the optimization problem would become:

$\underset{\mathbf{w}}{\operatorname{argmin}}\left\{\left\|\mathbf{e}_{N F}-\mathbf{S}_{N F} \cdot \mathbf{w}\right\|^{2}+\gamma\left\|\mathbf{e}_{F F}-\mathbf{S}_{F F} \cdot \mathbf{w}\right\|^{2}\right\}$

Using this methodology, different definitions of $f_{N F}(\mathbf{w})$ and $f_{F F}(\mathbf{w})$ lead to solving different problems accounting for both NF and FF specifications at the same time, weighting the two requirements by a proper choice of the trade-off parameter $\gamma$. Although above choice is highly dependent on the application and the criteria for choosing trade-off parameters are a typical issue of several optimization techniques, some steps are suggested to facilitate a proper selection. The solutions provided by the CP method for one-spot NFF are recognized as an effective starting point for the iterative algorithms (even for multiple spots, if a linear combination of the phase profiles for each point is used). Then, once it has been applied at the first step of the optimization, a quantitative information about the amplitude of each term of the cost function is obtained, and starting from this information we can decide the initial value to be assigned to the trade-off parameters. From then, an error and trail approach must be used. In the following sections, some significant examples and results will be presented to show the flexibility of the proposed technique.

\section{RESUlTS}

\section{A. Simulated results}

In this subsection, some simulated examples are considered so that the potential benefits of this procedure are shown.

In the first numerical example, the device to be powered is positioned in front of the antenna. It is set in the NF region, at a position defined by $\vec{r}=[0,0,4 \lambda]$ so that such position is specified as the target focal point. Additionally, a minimum waste of energy towards the FF region is desired, so FF specifications consist on a minimum FF radiation for the synthesized NFF array. Equation (8) is chosen for this problem, in order to minimize the variance of the FF pattern so that it is as omnidirectional as possible (namely, the power is equally distributed and radiation beams are avoided). The quasi-Newton optimization method [27] has been used to reach a solution. The array is chosen with the following parameters: $N=16 \times 16$ squared microstrip patches with both width and lenght equal to $0.5 \lambda_{d}$, where $\lambda_{d}$ is the wavelength in the dielectric material [28]. The 


\begin{tabular}{|c|c|c|c|}
\hline & NF+FF opt. & NF opt. & CP \\
\hline Spot length $(z)$ & $1.93 \lambda$ & $1.86 \lambda$ & $1.87 \lambda$ \\
Spot width $(x)$ & $0.6 \lambda$ & $0.6 \lambda$ & $0.6 \lambda$ \\
Max. position & $\{0,0,3.9 \lambda\}$ & $\{0,0,3.9 \lambda\}$ & $\{0,0,4.05 \lambda\}$ \\
Dist. max-focal & $0.1 \lambda$ & $0.1 \lambda$ & $0.05 \lambda$ \\
\hline
\end{tabular}

TABLE I: Example \#1. Results using NF and FF optimization, NF-only optimization and the $\mathrm{CP}$ (ConjugatePhase) method.

patches are uniformly distributed in a rectangular grid placed at the $z=0$ plane, centered at the origin, and with interelement distance equal to $0.7 \lambda$.

In order to create the matrices $\mathbf{S}_{N F}$ and $\mathbf{S}_{F F}$, the NF region has been sampled evaluating the field distributions at a cubic grid with points separated $0.5 \lambda$. The sampled region has been limited to $x \in[-10 \lambda, 10 \lambda]$, $y \in[-10 \lambda, 10 \lambda]$ and $z \in[0.5 \lambda, 20 \lambda]$. The FF region has been sampled in directions separated one degree, both in $\theta$ and $\phi$ angular coordinates, with $\theta$ changing between 0 and 90 degrees. The parameter $\gamma$ has been set to 100 after previous tests.

The main results are shown in Fig. 1 (normalized NF amplitude in the plane $y=0$ ) and Fig. 2 (normalized $\mathrm{NF}$ amplitude along the array axis, namely the $z$-axis). Some numerical parameters of the achieved focal spot are summarized in Table I. For comparison purposes, above results have been obtained by considering the proposed approach, as well as two other criteria: NF-only optimization (without considering FF-requirements) and the conventional Conjugate-Phase approach (CP) that cannot consider any other input parameter other than the assigned focal point location.

From results in Fig. 2, it can be noticed that, for an assigned field level at the focal spot (corresponding to OdB for all curves), the lowest FF level is achieved by the proposed solution. Indeed, the directivity using the proposed approach is $5.13(7.10 \mathrm{~dB})$, while using NFonly optimization it is $5.58(7.47 \mathrm{~dB})$, and using the $\mathrm{CP}$ method it is $6.41(8.06 \mathrm{~dB})$. By considering that the single patch has a directivity equal to $6.3 \mathrm{~dB}$, it is apparent that the proposed approach is able to get a directivity slightly above the directivity of a patch array with an almost constant array factor. The lowest limit of $3 \mathrm{~dB}$ corresponding to an array with uniform radiation pattern in one hemisphere cannot be reached as this would require an array factor with a high amplitude in the directions outside the patch main beam, with an excessive degradation of the NF performance. Moreover, it is worth noting that all methods guarantee a directivity much lower than the directivity of a conventional FFfocused array with uniform amplitude and phase distributions (the latter being more than $30 \mathrm{~dB}$ for the considered $16 \times 16$ array). Additionally, for an assigned normalized

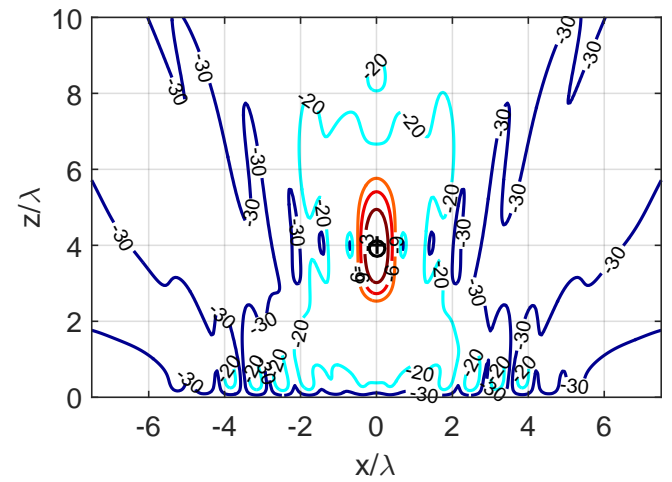

(a)

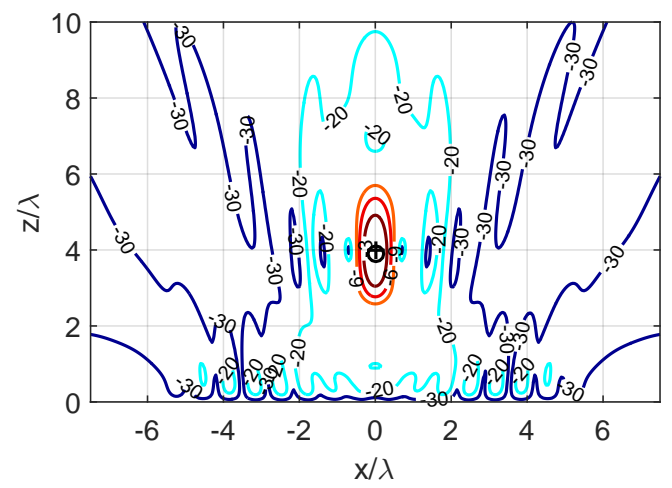

(b)

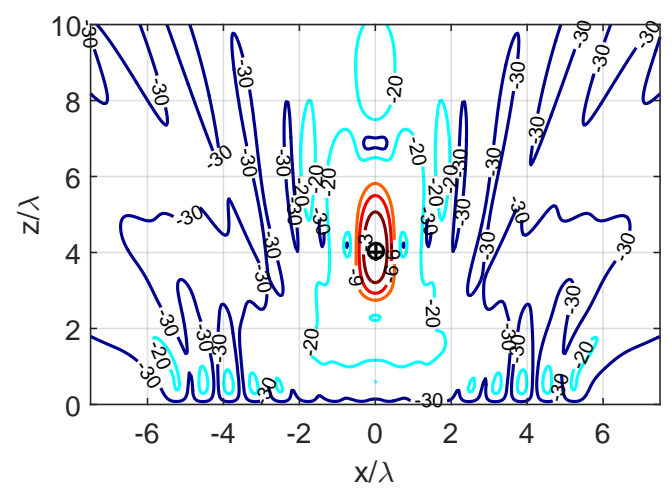

(c)

Fig. 1: Example \#1. Normalized Near Field distribution at $y=0$ for the NF+FF optimization scheme (a), the NF-only optimization (b) and the CP (Conjugate-Phase) method (c). The symbols + and $\circ$ represent the focal point and the synthesized field peak point, respectively.

radiated power density at the focal point $\left(1 \mathrm{~W} / \mathrm{m}^{2}\right)$, the total radiated power required by the proposed solution is only $1.31 \mathrm{~W}$, while the NF optimization and the CP solution require $1.33 \mathrm{~W}$ and $1.48 \mathrm{~W}$ respectively. In summary, the proposed approach ensures the lowest directivity and, for an assigned power density at the field peak, it is the 


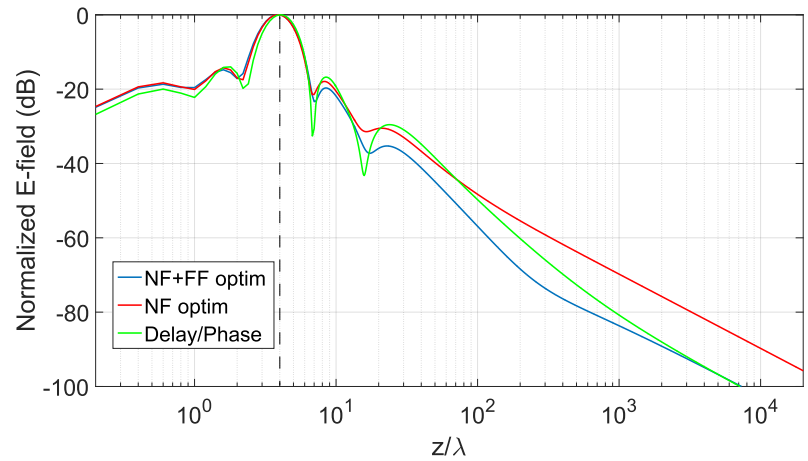

Fig. 2: Example \#1. Field level along the $z$ axis normalized to the level at the focal spot.

one requiring the lowest input power at the array feeding port.

It is interesting to point out that the case with a single focal point in front of the antenna produces a quite omnidirectional FF pattern independently of the method used to perform the synthesis. This fact is explained by the Theorem of Equivalence [29], which states that the focal spot may be viewed as an equivalent radiating source at the focal plane, and behaving as a quasiisotropic source towards the FF region as the spot width is smaller than the wavelength (see Table I). Under these circumstances it is impossible to obtain a more isotropic solution. Nonetheless, the use of the FF penalty term in the optimization scheme allows for an improvement in terms of both reduced directivity and less radiated power for an assigned power density at the focal spot, while NF performance is substantially not degraded with respect to the NF-only optimization.

Another interesting fact to be observed is the location of the maximum point provided by the $\mathrm{CP}$ solution, which is further away than the focal point instead of being closer to the antenna as it uses to be due to the focal shift effect [1]. It has been found that this phenomenon is due to the radiation pattern of the patches, especially because of the reduced contribution in the direction of the focal point from the elements placed at the array borders. The field contribution from these elements becomes more relevant at points at longer distance from the array surface, due to the typical broadside radiation pattern of patch antennas.

In a second example, a larger array has been considered in order to increase the NF performance. The number of elements is $N=32 \times 32$ patches, with the same properties as indicated for the first example. The distance between element centers is again $0.7 \lambda$. A focal point has been specified at $\vec{r}=[-5 \lambda, 0,13 \lambda]$. A FF main lobe is desired at the direction $\{\theta, \phi\}=\left\{45^{\circ}, 45^{\circ}\right\}$, and a radiation null is also specified at the direction $\{\theta, \phi\}=\left\{-15^{\circ}, 0\right\}$.

\begin{tabular}{|c|c|c|c|}
\hline & NF+FF opt. & NF opt. & CP \\
\hline Spot length $(z)$ & $3.2 \lambda$ & $3.04 \lambda$ & $3.14 \lambda$ \\
Spot width $(x)$ & $0.9 \lambda$ & $0.75 \lambda$ & $0.75 \lambda$ \\
Max. position & $\{-4.9,0,12.7\} \lambda$ & $\{-5,0,12.9\} \lambda$ & $\{-5,0,13\} \lambda$ \\
Dist. max-focal & $0.3 \lambda$ & $0.1 \lambda$ & 0 \\
\hline
\end{tabular}

TABLE II: Example \#2. Results using NF and FF optimization, NF-only optimization and the CP (ConjugatePhase) method.

The optimization problem has been defined as

$$
\begin{array}{r}
\underset{\mathbf{w}}{\operatorname{argmin}}\left\{\gamma_{N F}\left\|\mathbf{e}_{N F}-\mathbf{S}_{N F} \cdot \mathbf{w}\right\|^{2}+\gamma_{w}\|\mathbf{w}\|^{2}+\ldots\right. \\
\left.\ldots+\gamma_{F F}\left\|\mathbf{e}_{F F}-\mathbf{S}_{F F} \cdot \mathbf{w}\right\|^{2}\right\}
\end{array}
$$

where $\mathbf{e}_{N F}, \mathbf{S}_{N F}, \mathbf{e}_{F F}$ and $\mathbf{S}_{F F}$ are only specified or calculated for the positions and directions of interest; the first term accounts for the NF distribution, with $\mathbf{e}_{N F}=1$; the third term accounts for the FF pattern in the specified directions, with $\mathbf{e}_{F F}=[1,0]^{T}$ (the superscript $T$ standing for the transpose operator); the second term guarantees a minimum radiated power. It is worth noting that $\mathbf{e}_{N F}=1$ can be changed with any oher values different from unity, to control the difference between the power densities at the focal point and at any assigned location along the direction $\{\theta, \phi\}=\left\{45^{\circ}, 45^{\circ}\right\}$. Once more, the quasi-Newton method has been used for the minimization of the cost function. The use of this cost function that only accounts for the positions and directions of interest in the NF and FF regions allows not defining the grid used in the previous example or the set of spatial directions, hence reducing the computational requirements. The trade-off parameters used in the cost function have been set to $\gamma_{N F}=1, \gamma_{F F}=10$ and $\gamma_{w}=100$.

The NF distribution is represented in Fig. 3 for the three optimization methods. The quantitative results are summarized in Table II.

The field level along the axis passing through the focal point is represented in Fig. 4. Both Table II and Fig. 4 show that the CP method provides the best solution in terms of focusing at one point. However, the proposed method tries to achieve a solution combining both NF and FF performance. The NF performance is shown to be worse but reasonably good while the solution is also accounting for the FF specifications. Figure 5 shows the UV FF pattern where the specified lobe may be observed as well as the desired null.

\section{B. Prototype and measured results}

In order to extend the analysis of the validity of the present method, a prototype consisting of a $16 \mathrm{GHz}$ $16 \times 16$ planar microstrip array has been designed, manufactured and measured. The array is assumed to be placed in the plane $z=0$. Two focal points at $\{0,0,7\} \lambda$ 


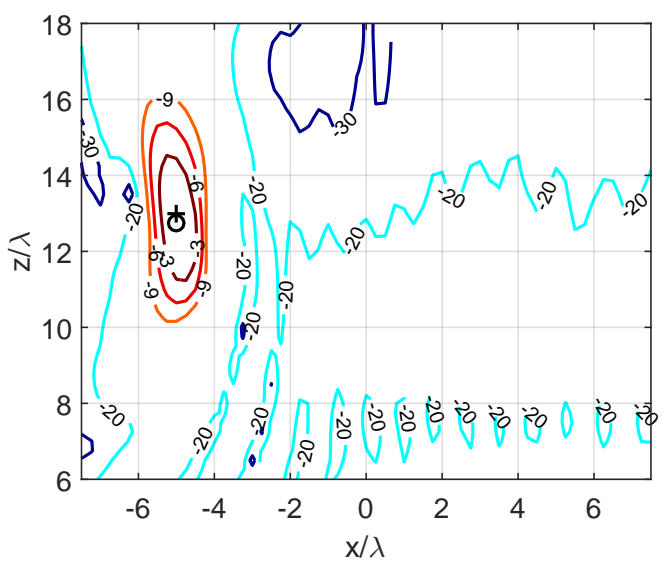

(a)

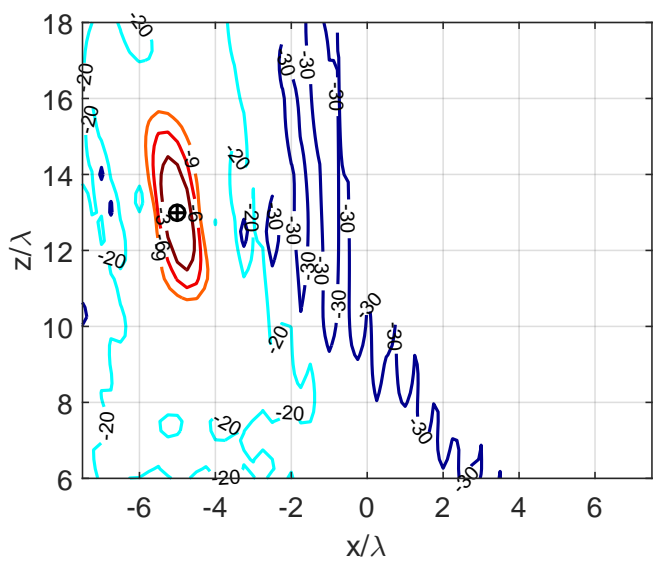

(b)

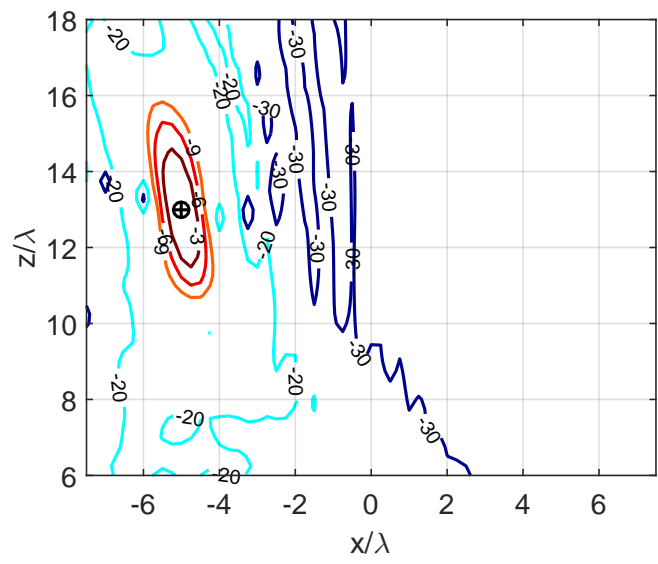

(c)

Fig. 3: Example \#2. Normalized Near Field distribution at $y=0$ for the NF+FF optimization scheme (a), the NF-only optimization (b) and the CP method (c). The symbols + and $\circ$ represent the focal and synthesized maximum points respectively.

and $\{5.75,0,6\} \lambda$, and a FF-main lobe at $\{\theta, \phi\}=$

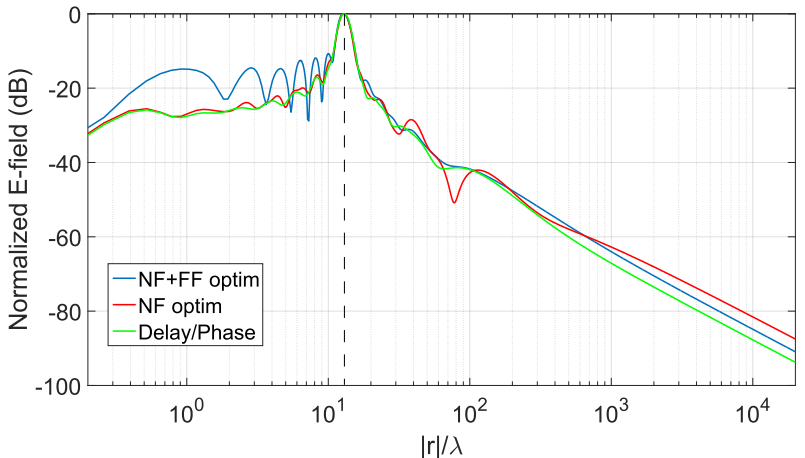

Fig. 4: Example \#2. Field level across the axis through the focal point normalized to the level at the focal spot.

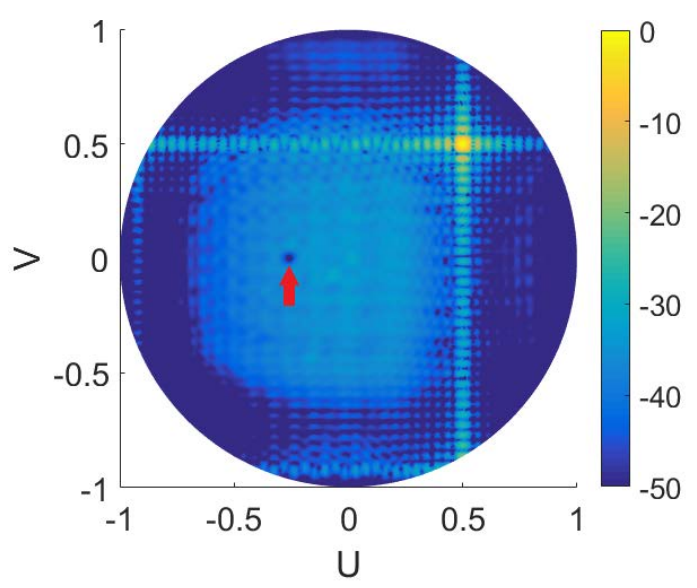

Fig. 5: Example \#2. Normalized UV FF pattern for the NF+FF optimization scheme. The null position is indicated with a red arrow.

$\left\{45^{\circ}, 45^{\circ}\right\}$ have been specified simultaneously. The array elements are microstrip square patches of $5.2 \mathrm{~mm}$ of side, each coupled by a rectangular slot $(4.6 \mathrm{~mm} \times 0.4 \mathrm{~mm}$ wide) and fed by a $100 \Omega$ microstrip line. These lines are connected through a typical $50 \Omega$ T-junction. Both patches and feeding network have been manufactured on a Rogers 4003 substrate with a thickness of $0.41 \mathrm{~mm}$ (16 mils). The slots and the patches are separated by an air layer of $1.4 \mathrm{~mm}$. The distance between array elements is $0.7 \lambda$. To get a $2 \pi$ phase shifts, each patch is fed by an U-bend that can be tuned to achieve a 180 degree phase shift, while the remaining 180 degrees can be obtained by feeding the opposite side of the patch. In Fig. 6, the developed feeding network (Fig. 6a) and a detail of it (Fig. 6b) may be observed. Equation 9 has been used to calculate the weights that must be applied to the radiating elements, with the constraint $\left|w_{n}\right|=1, \forall n=1 \ldots N$, so that only the phase of the weights has to be optimized. The interior-point optimization method [27] has been used to reach the solution, with the trade-off parameter 


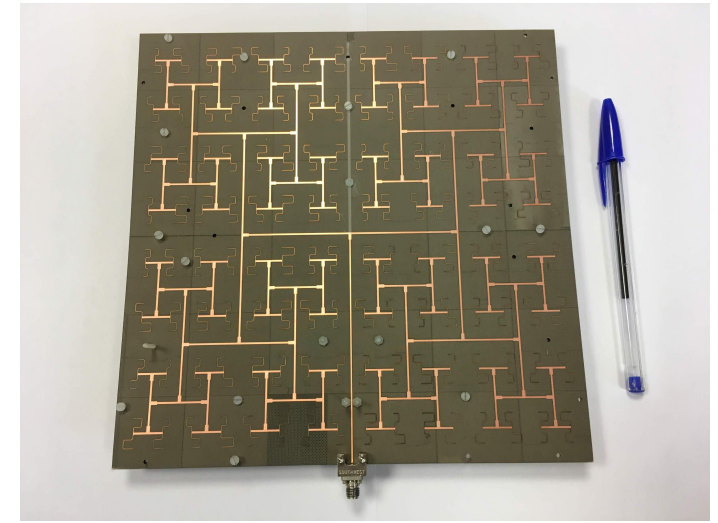

(a)

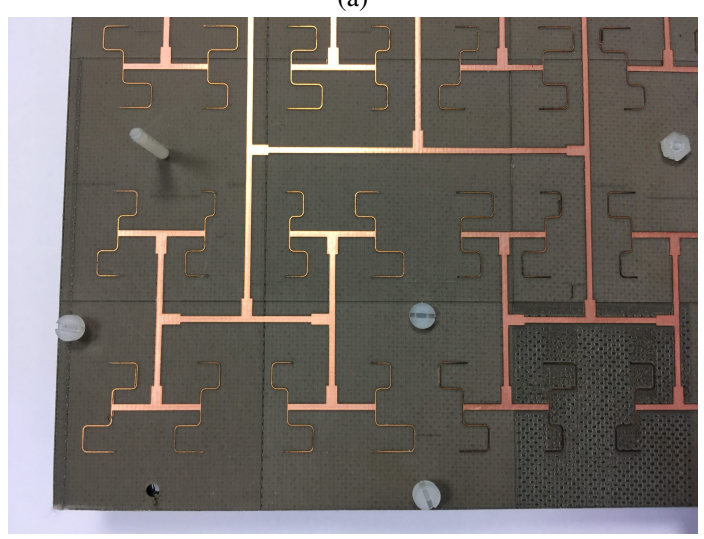

(b)

Fig. 6: Prototyped antenna feeding network (a) and a detailed view of some feeding lines (b).

set to $\gamma=0.1$.

The FF radiation pattern and near field have been measured in the anechoic chamber (spherical range) and the anechoic planar scanner, respectively, at the measurement facilities of the Group of Signal Theory and Communications of the University of Oviedo. For NF measurements, the field distribution was studied at the target planes $z=6 \lambda$ and $z=7 \lambda$, at the plane $y=0$ and at the planes $x=0$ and $x=5.75 \lambda$, so that the target points and the surrounding spots may be clearly identified.

The comparison between simulated and measured results is represented in Figs. 7, 8, 9, 10, 11 and 12. Although some unexpected lobes or spots are present all over the environment (specially visible in the $y=0$ plane), there is a good agreement between the simulated and measured positions of the main spots and the resulting FF pattern. The differences are due to some manufacturing inaccuracies.

The measured focusing parameters are summarized in Table III. It is curious that the prototype generates two maxima closer to the focal points than the simulated

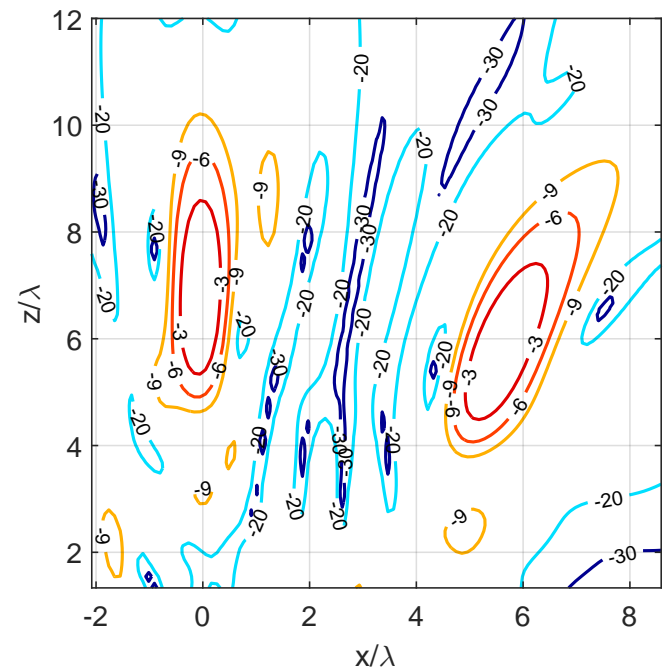

(a)

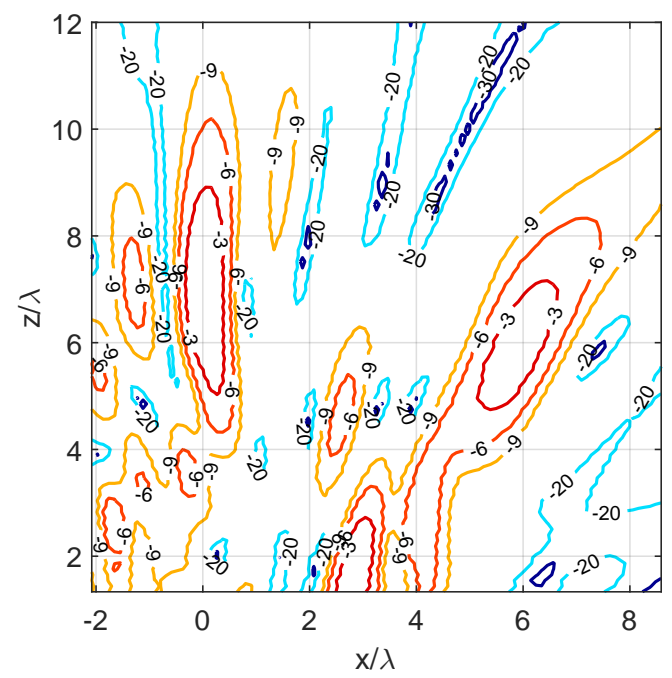

(b)

Fig. 7: Prototype. Normalized NF distribution at $y=0$, simulated (a) and measured (b).

ones.

\section{Example of application: 5G WPT scenario}

A possible future WPT scenario at a working frequency of $28 \mathrm{GHz}$ (a typical $5 \mathrm{G}$ case) is considered in this section, where an illustrative NF area that might be of interest in many cases is studied. Different spotsize may be necessary according to the devices involved: for example, small spots might be interesting for wireless sensors and larger areas may be requested to give coverage to laptops or similar-sized devices, meanwhile allowing having FF radiation in a given direction to allow connectivity between femto-cells and the rest of $5 \mathrm{G}$ network, and optimizing energy allocation at the 


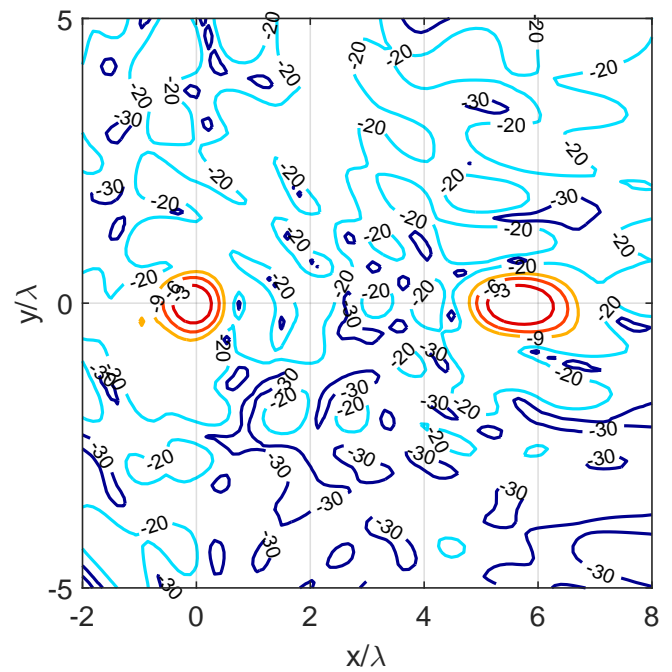

(a)

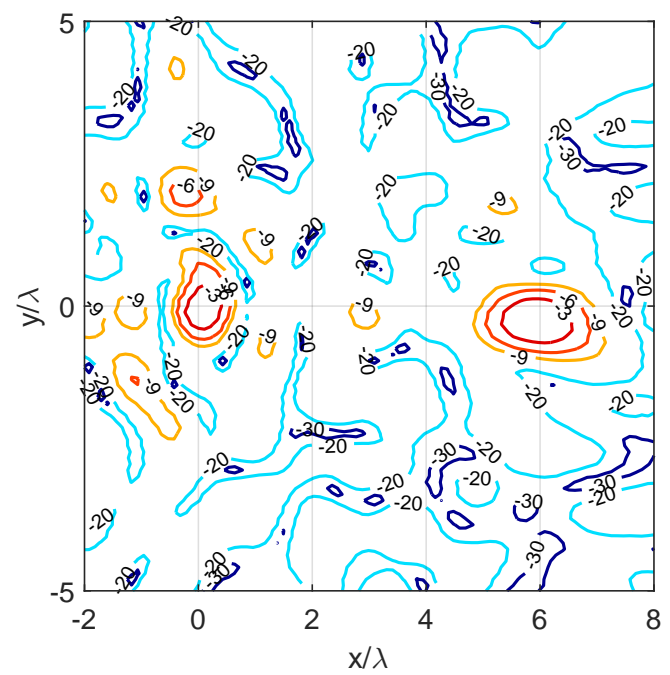

(b)

Fig. 8: Prototype. Normalized NF distribution at $z=6 \lambda$, simulated (a) and measured (b).

same time. In order to illustrate such situation, a uniform array with $N=32 \times 32$ elements and an interelement distance equal to $0.6 \lambda$ is considered (other electrical sizes are as for the first example). At $28 \mathrm{GHz}$, a realistic distance between the antenna and a device to be powered might be $200 \lambda=2.14 \mathrm{~m}$, which represents a location in the array NF region as the FF region is located beyond a distance of $10 \mathrm{~m}$ according to the definition given by $2 D^{2} / \lambda$ [30], being $D$ the size of the antenna. For this example, let us assume that a device to be powered is located at $\vec{r}=[-20 \lambda, 0,200 \lambda]$; in order to create a more realistic application, the focal spot is required to be, at least, $10 \mathrm{~cm} \times 10 \mathrm{~cm}$ wide. Another device to be powered or another element of the $5 \mathrm{G}$ network is

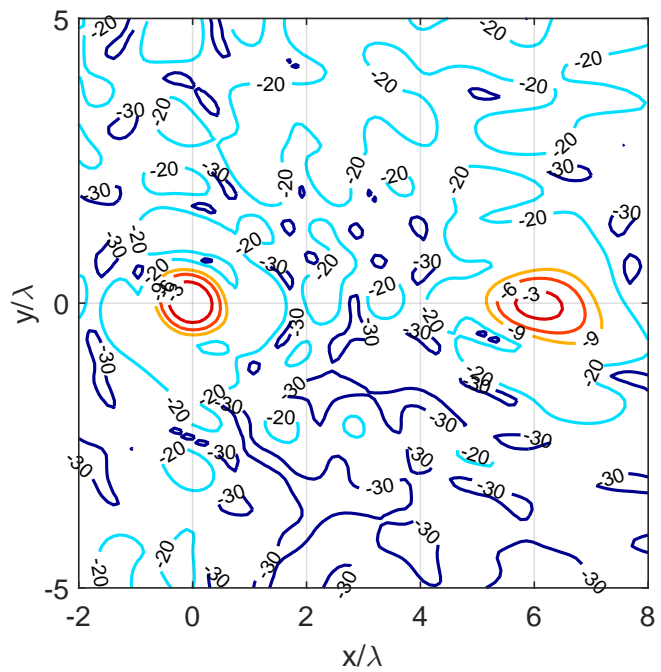

(a)

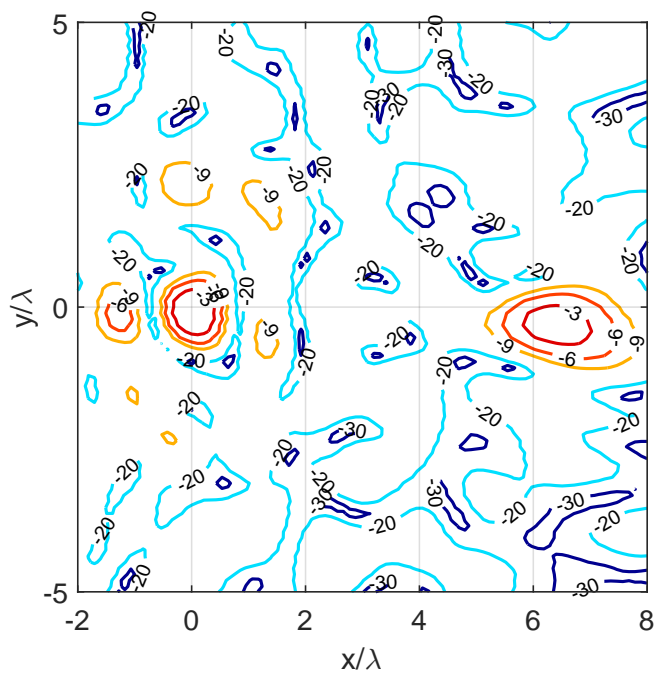

(b)

Fig. 9: Prototype. Normalized NF distribution at $z=7 \lambda$, simulated (a) and measured (b).

placed at a position in the FF-region, along the direction $\{\theta, \phi\}=\left\{45^{\circ}, 60^{\circ}\right\}$; additionally, a sensitive device that should not receive radiation may be located in the FFregion at an unpredictable direction, so the overall FF radiation should be reduced.

The optimization problem for this case is set to

$$
\begin{aligned}
& \operatorname{argmin}\left\{\gamma_{N F}\left\|\tilde{\mathbf{S}}_{N F} \cdot \mathbf{w}-\mathbf{1}^{T}\right\|^{2}+\gamma_{w}\|\mathbf{w}\|^{2}+\ldots\right. \\
& \left.\ldots+\gamma_{F F}\left\|\tilde{\mathbf{S}}_{F F} \cdot \mathbf{w}-1\right\|^{2}\right\}
\end{aligned}
$$

where $\tilde{\mathbf{S}}_{F F}$ is a reduced version of $\mathbf{S}_{F F}$ accounting only for the specified direction of the space, in the same way as $\tilde{\mathbf{S}}_{N F}$ is related to $\mathbf{S}_{N F}$. In order to create the spot with the specified dimensions in the NF region, the targeted 


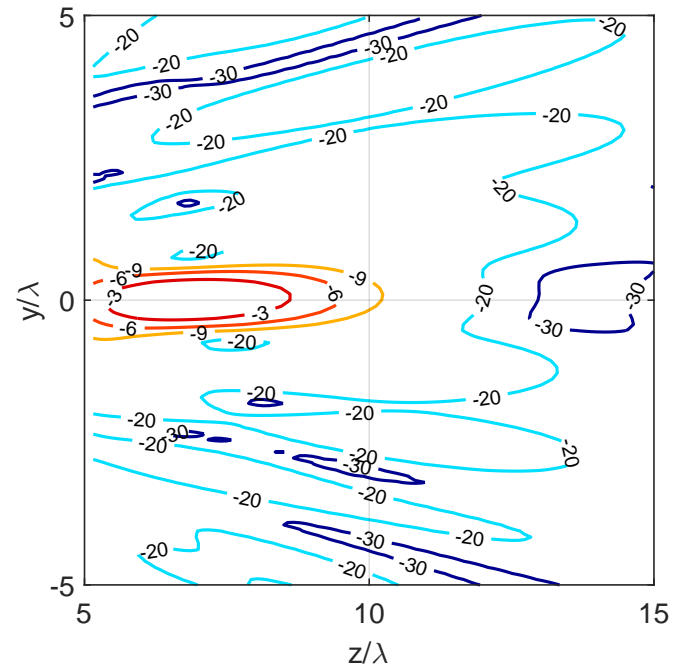

(a)

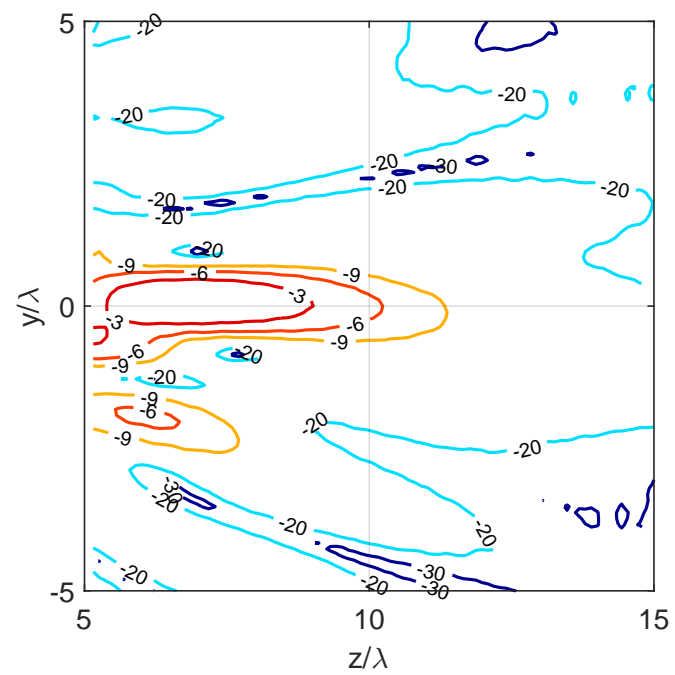

(b)

Fig. 10: Prototype. Normalized NF distribution at $x=0$, simulated (a) and measured (b).

normalized field at a set of positions at $\pm 10 \lambda$ from the focal point has been set to 1 , so that these values are arranged in the vector $\mathbf{1}=[1,1, \ldots]$. In this way, a wider focal spot is enforced. The trade-off parameters have been set to $\gamma_{N F}=0.2, \gamma_{w}=100$ and $\gamma_{F F}=500$. No constraints have been included, and the quasi-Newton method has been used to solve the optimization problem.

The NF distribution has been represented for the plane $z=200 \lambda$ in Fig. 13, where a clear $-3 \mathrm{~dB}$ spot of about $17.5 \times 14 \mathrm{~cm}$ is identified ( $x$ and $y$ respectively). The FF UV pattern is plotted in Fig. 14, where the desired lobe is clearly identified, and the overall FF radiation is low. It is worth noting a peak of the field at the FF-direction close to the broadside direction; it corresponds to the

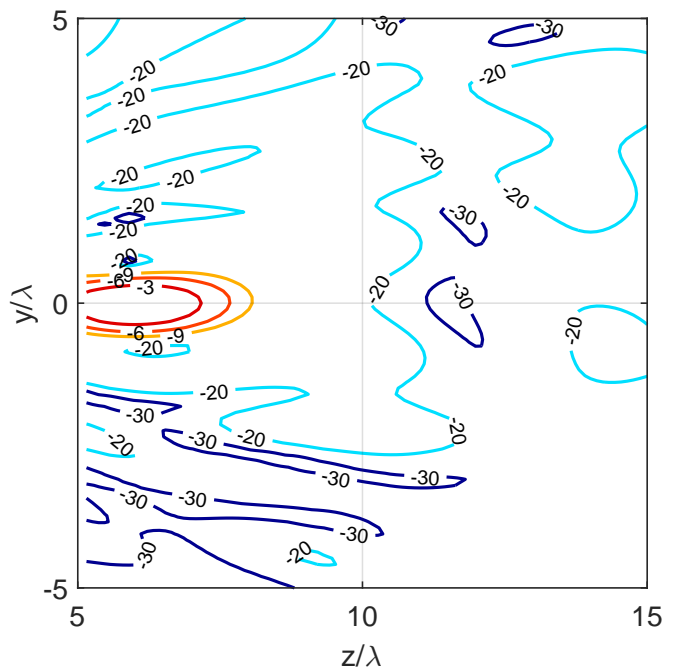

(a)

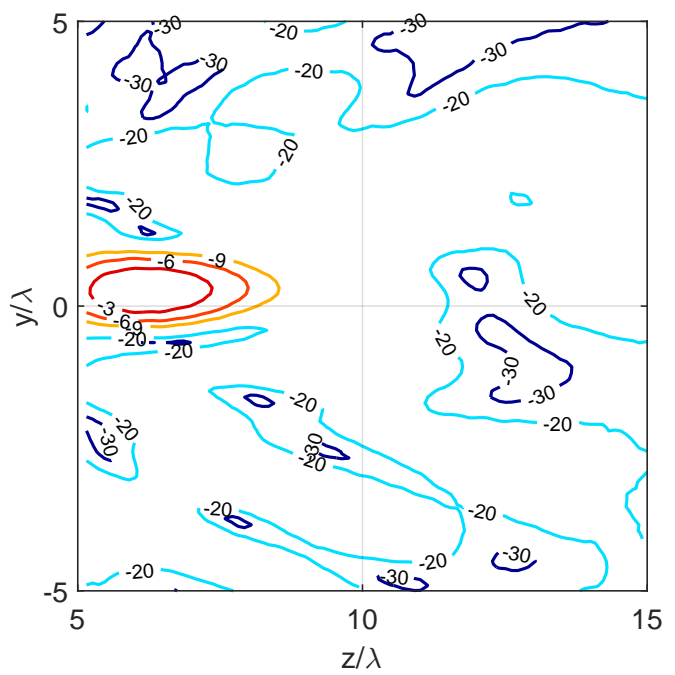

(b)

Fig. 11: Prototype. Normalized NF distribution at $x=$ $5.75 \lambda$, simulated (a) and measured (b).

end of the focal spot at the opposite side with respect to the array surface.

As the results show, the proposed method allows generating an area at the given distance so that energy wasted in other positions is reduced, simultaneously accounting for FF specifications. However, it has been observed that the maximum of radiation in the nearfield is closer to the antenna. The specification of a broad spot in the plane $z=200 \lambda$ enforces a wide lobe towards it, hence reducing the constructive interference in the areas closer to the plane of interest. If a smaller spot is specified, the lobe is allowed to be narrower displacing the maximum point towards the specified plane. In any case, it is worth noticing that the specified 


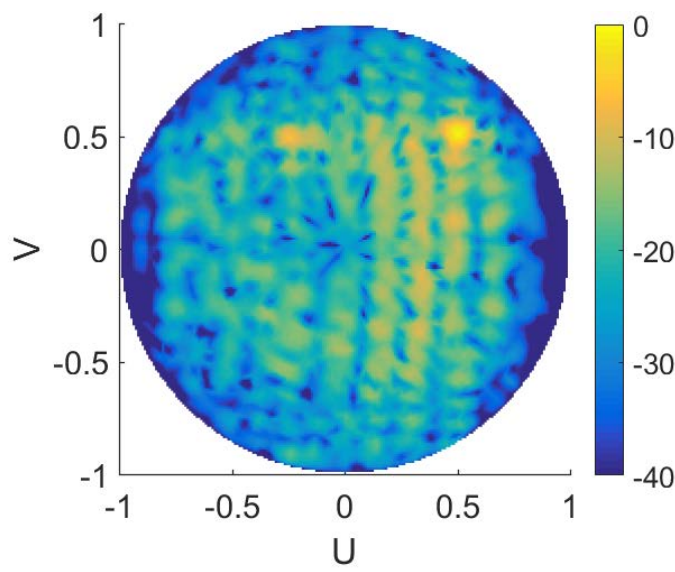

(a)

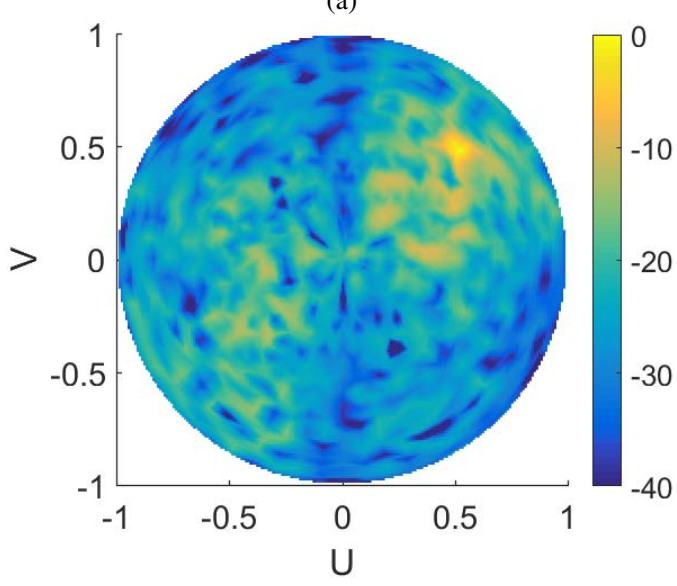

(b)

Fig. 12: Prototype. Normalized FF pattern, simulated (a) and measured (b). The specifications required a FF main beam at the direction $\{\theta, \phi\}=\left\{45^{\circ}, 45^{\circ}\right\}$ (corresponding to $U=0.5$ and $V=0.5$ ).

\begin{tabular}{|c|c|c|}
\hline & Simulated & Measured \\
\hline Spot 1 length $(z)$ & $3.25 \lambda$ & $3.8 \lambda$ \\
Spot 1 width $(x)$ & $0.74 \lambda$ & $0.68 \lambda$ \\
Max. position 1 & $\{0.05,0,6.67\} \lambda$ & $\{0.05,0,6.77\} \lambda$ \\
Dist. max-focal 1 & $0.33 \lambda$ & $0.23 \lambda$ \\
\hline Spot 2 length $(z)$ & $3.15 \lambda$ & $2.7 \lambda$ \\
Spot 2 width (x) & $1.04 \lambda$ & $0.85 \lambda$ \\
Max. position 2 & $\{5.49,0,5.6\} \lambda$ & $\{5.7,0,5.6\} \lambda$ \\
Dist. max-focal 2 & $0.47 \lambda$ & $0.4 \lambda$ \\
\hline
\end{tabular}

TABLE III: Prototype. Simulated and measured parameters of the focal spots.

problem does not require a conventional focal point to be specified at the plane $z=200 \lambda$. Moreover, the position of the field maximum is a consequence of the size of the defined spot and the distance between it and the array. Other definitions of the cost function may be used if further constraints regarding focusing maxima are necessary, probably leading to a trade-off between

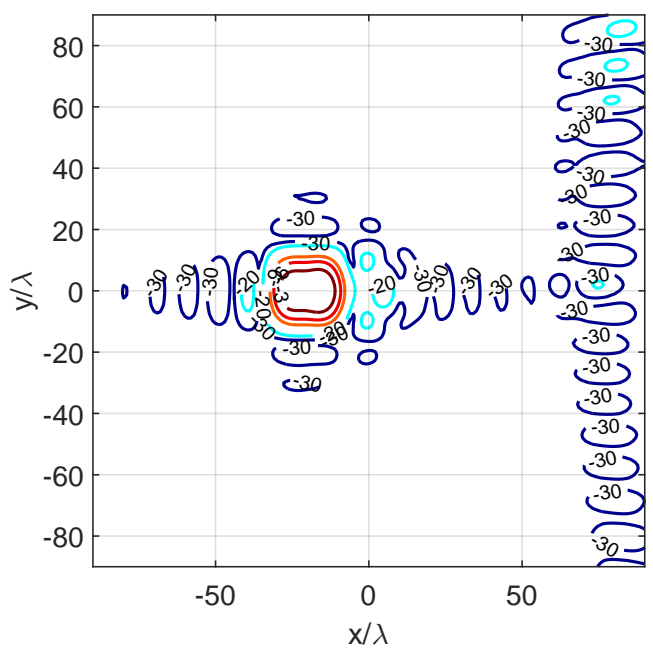

(a)

Fig. 13: 5G example. Near Field distribution at $z=$ $200 \lambda$.

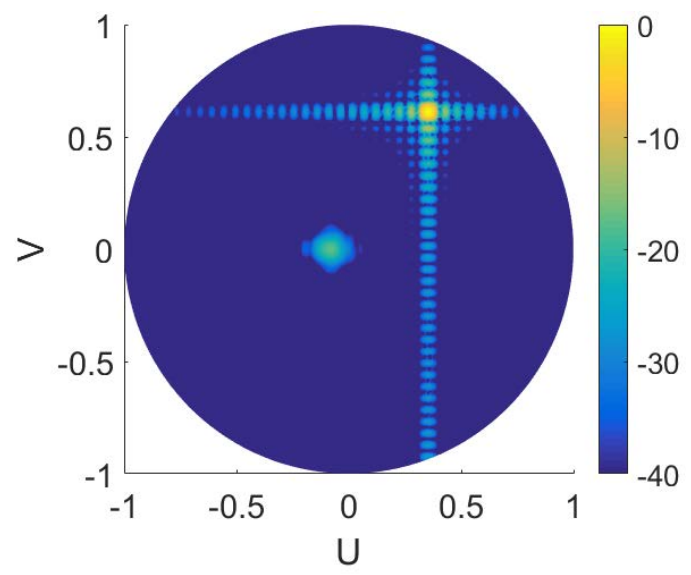

(a)

Fig. 14: 5G example. FF UV pattern. The specifications required a FF main beam at the direction $\{\theta, \phi\}=$ $\left\{45^{\circ}, 60^{\circ}\right\}$ (corresponding to $U=0.35$ and $V=0.61$ ).

spot size, position and field amplitude.

\section{CONCLUSION}

A quite general array synthesis technique able to account, simultaneously, for both near-field and far-field requirements has been introduced. Its effectiveness has been proved in different scenarios. An acceptable degradation of NF performance is paid when FF specifications are added, however allowing for a number of advantages: less energy distributed towards the FF region, a main lobe directed towards an assigned FF target, combination of lobes and nulls at assigned FF directions, shaping 
of the NF focal spot. The proposed array synthesis technique opens the door to the solution of complex problems where field requirements in almost any location around the antenna may be specified.

The design, development and measurement of a prototype that accounts for both NF and FF specifications at the same time support the presented synthesis technique, as well as a potential future 5G scenario where WPT is carried out over devices placed at both NF and FF positions, while keeping aside high-sensitivity devices that should not receive radiation.

Regarding the methodology, different cost functions or optimization schemes may be used, which leads to an extremely flexible procedure able to solve for many different problems. In those optimization schemes requiring for an effective starting solution, the phase profile obtained by simply applying the conventional conjugate-phase approach can improve the convergence performance of the whole synthesis problem.

\section{REFERENCES}

[1] R. Hansen, "Focal region characteristics of focused array antennas," IEEE Transactions on Antennas and Propagation, vol. 33, no. 12, pp. 1328-1337, December 1985.

[2] A. Buffi, P. Nepa, and G. Manara, "Design criteria for nearfield-focused planar arrays," IEEE Antennas and Propagation Magazine, vol. 54, no. 1, pp. 40-50, Feb 2012.

[3] P. Nepa and A. Buffi, "Near-field-focused microwave antennas: Near-field shaping and implementation." IEEE Antennas and Propagation Magazine, vol. 59, no. 3, pp. 42-53, June 2017.

[4] P. Nepa, A. Buffi, A. Michel, and G. Manara, "Technologies for near-field focused microwave antennas," International Journal of Antennas and Propagation, vol. 2017, p. 17 pages, 2017.

[5] W. Gee, S. W. Lee, N. K. Bong, C. A. Cain, R. Mittra, and R. L. Magin, "Focused array hyperthermia applicator: Theory and experiment," IEEE Transactions on Biomedical Engineering, vol. BME-31, no. 1, pp. 38-46, Jan 1984.

[6] K. H. Sayidmarie and A. M. Abdulkhaleq, "Investigation of six array geometries for focused array hyperthermia applications," Progress In Electromagnetics Research M, vol. 23, pp. 181-194, 2012.

[7] X. He, W. Geyi, and S. Wang, "Optimal design of focused arrays for microwave-induced hyperthermia," IET Microwaves, Antennas Propagation, vol. 9, no. 14, pp. 1605-1611, 2015.

[8] M. Bogosanovic and A. G. Williamson, "Microstrip antenna array with a beam focused in the near-field zone for application in noncontact microwave industrial inspection," IEEE Transactions on Instrumentation and Measurement, vol. 56, no. 6, pp. 2186 2195, Dec 2007.

[9] A. Buffi, A. A. Serra, P. Nepa, H. T. Chou, and G. Manara, "A focused planar microstrip array for $2.4 \mathrm{GHz}$ RFID readers," IEEE Transactions on Antennas and Propagation, vol. 58, no. 5, pp. 1536-1544, May 2010.

[10] H. T. Chou, T. M. Hung, N. N. Wang, H. H. Chou, C. Tung, and P. Nepa, "Design of a near-field focused reflectarray antenna for 2.4GHz RFID reader applications," IEEE Transactions on Antennas and Propagation, vol. 59, no. 3, pp. 1013-1018, March 2011.

[11] H. T. Chou, M. Y. Lee, and C. T. Yu, "Subsystem of phased array antennas with adaptive beam steering in the near-field RFID applications," IEEE Antennas and Wireless Propagation Letters, vol. 14, pp. 1746-1749, 2015.
[12] R. Zhang and C. K. Ho, "Mimo broadcasting for simultaneous wireless information and power transfer," IEEE Transactions on Wireless Communications, vol. 12, no. 5, pp. 1989-2001, May 2013.

[13] S. Yu, H. Liu, and L. Li, "Design of near-field focused metasurface for high efficient wireless power transfer with multi-focus characteristics," IEEE Transactions on Industrial Electronics, pp. $1-1,2018$.

[14] G. Monti, A. Costanzo, F. Mastri, M. Mongiardo, and L. Tarricone, "Rigorous design of matched wireless power transfer links based on inductive coupling," Radio Science, vol. 51, no. 6, pp. 858-867, June 2016.

[15] A. Costanzo and D. Masotti, "Smart solutions in smart spaces: Getting the most from far-field wireless power transfer," IEEE Microwave Magazine, vol. 17, no. 5, pp. 30-45, May 2016.

[16] J. L. Gomez-Tornero, A. R. Weily, and Y. J. Guo, "Rectilinear leaky-wave antennas with broad beam patterns using hybrid printed-circuit waveguides," IEEE Transactions on Antennas and Propagation, vol. 59, no. 11, pp. 3999-4007, Nov 2011.

[17] A. J. Martínez-Ros, J. L. Gómez-Tornero, F. J. ClementeFernández, and J. Monzó-Cabrera, "Microwave near-field focusing properties of width-tapered microstrip leaky-wave antenna," IEEE Transactions on Antennas and Propagation, vol. 61, no. 6, pp. 2981-2990, June 2013.

[18] R. G. Ayestarán, "Fast near-field multifocusing of antenna arrays including element coupling using neural networks," IEEE Antennas and Wireless Propagation Letters, vol. 17, no. 7, pp. 1233-1237, July 2018.

[19] J. Alvarez, R. G. Ayestaran, G. Leon, L. F. Herran, A. Arboleya, J. A. Lopez-Fernandez, and F. Las-Heras, "Near field multifocusing on antenna arrays via non-convex optimisation," IET Microwaves, Antennas Propagation, vol. 8, no. 10, pp. 754-764, July 2014.

[20] J. Alvarez, R. G. Ayestaran, and F. Las-Heras, "Design of antenna arrays for near-field focusing requirements using optimisation," Electronics Letters, vol. 48, no. 21, pp. 1323-1325, October 2012.

[21] D. A. M. Lero, L. Crocco, and T. Isernia, "Advances in 3-d electromagnetic focusing: Optimized time reversal and optimal constrained power focusing," Radio Science, vol. 52, no. 1, pp. 166-175, Jan 2017.

[22] G. G. Bellizzi, D. A. M. Iero, L. Crocco, and T. Isernia, "Threedimensional field intensity shaping: The scalar case," IEEE Antennas and Wireless Propagation Letters, vol. 17, no. 3, pp. 360-363, March 2018.

[23] G. G. Bellizzi, M. T. Bevacqua, L. Crocco, and T. Isernia, "3-d field intensity shaping via optimized multi-target time reversal," IEEE Transactions on Antennas and Propagation, vol. 66, no. 8, pp. 4380-4385, Aug 2018.

[24] X. Wang and C. Zhai, "Simultaneous Wireless Information and Power Transfer for Downlink Multi-User Massive Antenna-Array Systems," IEEE Transactions on Communications, vol. 65, no. 9, pp. 4039-4048, Sep 2017.

[25] W. Hong, Z. H. Jiang, C. Yu, J. Zhou, P. Chen, Z. Yu, H. Zhang, B. Yang, X. Pang, M. Jiang, Y. Cheng, M. K. T. Al-Nuaimi, Y. Zhang, J. Chen, and S. He, "Multibeam Antenna Technologies for 5G Wireless Communications," IEEE Transactions on Antennas and Propagation, vol. 65, no. 12, pp. 6231-6249, Dec 2017.

[26] Y. Cao, K. Chin, W. Che, W. Yang, and E. S. Li, "A Compact 38 $\mathrm{GHz}$ Multibeam Antenna Array With Multifolded Butler Matrix for 5G Applications," IEEE Antennas and Wireless Propagation Letters, vol. 16, pp. 2996-2999, 2017.

[27] J. Bonnans, J. Gilbert, C. Lemarechal, and C. Sagastizábal, Numerical Optimization: Theoretical and Practical Aspects, ser. Universitext. Springer Berlin Heidelberg, 2013.

[28] W. Stutzman and G. Thiele, Antenna Theory and Design, 3rd Edition, ser. Antenna Theory and Design. Wiley, 2012.

[29] R. F. Harrington, Time-Harmonic Electromagnetic Fields. IEEEPress, 2001

[30] C. A. Balanis, Antenna Theory: Analysis and Design. New York, NY, USA: Wiley-Interscience, 2005. 